\title{
New NIR spectro-polarimetric modes for the SCEXAO instrument
}

Lozi, Julien, Guyon, Olivier, Kudo, Tomoyuki, Zhang, Jin, Jovanovic, Nemanja, et al.

Julien Lozi, Olivier Guyon, Tomoyuki Kudo, Jin Zhang, Nemanja Jovanovic, Barnaby Norris, Marc-Antoine Martinod, Tyler D. Groff, Jeffrey Chilcote, Motohide Tamura, Steven Bos, Frans Snik, Sébastien Vievard, Ananya Sahoo, Vincent Deo, Frantz Martinache, Jeremy Kasdin, "New NIR spectropolarimetric modes for the SCEXAO instrument," Proc. SPIE 11448, Adaptive Optics Systems VII, 114487C (13 December 2020); doi: 10.1117/12.2562792 


\title{
New NIR spectro-polarimetric modes for the SCExAO instrument
}

Julien Lozi ${ }^{\mathrm{a}}$, Olivier Guyon ${ }^{\mathrm{a}, \mathrm{b}, \mathrm{c}, \mathrm{d}}$, Tomoyuki Kudo ${ }^{\mathrm{a}, \mathrm{d}}$, Jin Zhang ${ }^{\mathrm{e}}$, Nemanja Jovanovic ${ }^{\mathrm{f}}$,

Barnaby Norris ${ }^{\mathrm{g}}$, Marc-Antoine Martinod ${ }^{\mathrm{g}}$, Tyler D. Groff ${ }^{\mathrm{h}}$, Jeffrey Chilcote ${ }^{\mathrm{i}}$, Motohide Tamura $^{\mathrm{d}, \mathrm{e}, \mathrm{j}}$, Steven Bos ${ }^{\mathrm{k}}$, Frans Snik ${ }^{\mathrm{k}}$, S'ebastien Vievard ${ }^{\mathrm{a}, \mathrm{d}, \mathrm{l}}$, Ananya Sahoo ${ }^{\mathrm{a}, \mathrm{m}}$, Vincent Deo ${ }^{\mathrm{a}}$, Frantz Martinache ${ }^{\mathrm{n}}$, and Jeremy Kasdin ${ }^{\circ}$

a Subaru Telescope, National Astronomical Observatory of Japan, National Institutes of Natural Sciences (NINS), 650 North A‘ohōkū Place, Hilo, HI, 96720, U.S.A.

${ }^{\mathrm{b} S t e w a r d ~ O b s e r v a t o r y, ~ U n i v e r s i t y ~ o f ~ A r i z o n a, ~ T u c s o n, ~ A Z, ~ 85721, ~ U . S . A . ~}$

${ }^{\mathrm{c}}$ College of Optical Sciences, University of Arizona, Tucson, AZ 85721, U.S.A.

${ }^{\mathrm{d} A s t r o b i o l o g y}$ Center of NINS, 2-21-1, Osawa, Mitaka, Tokyo, 181-8588, Japan

e'University of Tokyo, 7 Chome-3-1 Hongo, Bunkyo City, Tokyo 113-8654, Japan

${ }^{\mathrm{f}}$ California Institute of Technology, 1200 E California Blvd, Pasadena, CA 91125, U.S.A.

${ }^{g}$ Sydney Institute for Astronomy, Institute for Photonics and Optical Science, School of

Physics, University of Sydney, NSW 2006, Australia

${ }^{\mathrm{h}}$ Goddard Space Flight Center, 8800 Greenbelt Rd, Greenbelt, MD 20771, U.S.A

${ }^{i}$ Univeristy of Notre Dame, Notre Dame, IN 46556, U.S.A.

${ }^{j}$ National Astronomical Observatory of Japan, National Institutes of Natural Sciences (NINS),

2 Chome-21-1 Osawa, Mitaka, Tokyo 181-0015, Japan

${ }^{k}$ Leiden Observatory, Leiden University, P.O. Box 9513, 2300 RA Leiden, The Netherlands

${ }^{1}$ Observatoire de Paris, LESIA, 5 Place Jules Janssen, 92190 Meudon, France

${ }^{m}$ Sokendai, Graduate University for Advanced Studies, Kanagawa Prefecture, Miura District,

Hayama, Shonankokusaimura, 240-0193, Japan

${ }^{n}$ Laboratoire Lagrange, Université Côte d'Azur, Observatoire de la Cô]te d'Azur, CNRS, Parc

Valrose, Bât. H. FIZEAU, 06108 Nice, France

${ }^{\circ}$ Princeton University, Princeton, NJ 08544, U.S.A.

\begin{abstract}
Polarization Differential Imaging (PDI) is one of the most productive modes of current high-contrast imagers. Dozens of new protoplanetary, transition and debris disks were imaged recently for the first time, helping us understand the processes of planet formation, and giving clues on the mass of potential planets inside these disks, even if they cannot be imaged directly. The Subaru Coronagraphic Extreme Adaptive Optics (SCExAO) instrument is equipped with a fast visible dual-camera polarimetric module, VAMPIRES, already producing valuable scientific observations of protoplanetary disks and dust shells. In addition, we recently commissioned two new polarimetric modules in the infrared. The first one is a spectro-polarimetric mode using the CHARIS Integral Field Spectrograph (IFS). A Wollaston prism was added in front of the IFS, reducing the field-of-view to $2 \mathrm{x} 1$ arcsec to accommodate for the imaging of both polarizations on the same detector without sacrificing the spectral resolution of the instrument, in any of its spectral modes. The second module, similar to VAMPIRES, uses a low-noise high frame rate C-RED ONE camera combined with a Ferroelectric Liquid Crystal (FLC) device to modulate and record the polarization at high-speed, freezing effectively the atmospheric speckles for higher precision. We present on-sky results of the new polarimetric capabilities taken during the commissioning phase. In addition, we show future capabilities that are already scheduled to increase the performance of these modules,
\end{abstract}

Further author information: (Send correspondence to J.L.)

J.L.: E-mail: lozi@naoj.org, Telephone: 18089345949

Adaptive Optics Systems VII, edited by Laura Schreiber, Dirk Schmidt, Elise Vernet, Proc. of SPIE Vol. 11448, 114487C · C 2020 SPIE · CCC code: 0277-786X/20/\$21 - doi: 10.1117/12.2562792 
especially the addition of non-redundant masks, as well as a polarimetric vector Apodizing Phase Plate (vAPP) coronagraph.

Keywords: high-contrast imaging, polarimetry, spectro-polarimetry, adaptive optics

\section{INTRODUCTION}

The successful recent campaigns looking for protoplanetary disks, transition disks and debris disks with polarimetric modes of $\mathrm{SPHERE}^{1}$ and $\mathrm{GPI}^{2}$ demonstrate the power of polarization differential imaging (PDI) for high-contrast instruments. Observing these disks, especially combined with ALMA observations in the submillimeter, reveals processes of planet formations, and can even indicate the presence of planets despite being too small to be observed with traditional high-contrast imaging methods. PDI was an important component of the High Contrast Instrument for the Subaru Next Generation Adaptive Optics (HiCIAO), ${ }^{3}$ and produced high-contrast images of about a dozen new disks. ${ }^{4}$ It's successor, the Subaru Coronagraphic Extreme Adaptive Optics (SCExAO), ${ }^{5}$ is a high-contrast instrument capable of extreme wavefront correction, to achieve deeper contrasts. Its modular design allows to add new capabilities easily, and validate them in the lab and on-sky in a short time ${ }^{6}$ (see Lozi et al. from this conference). SCExAO was equipped with the VAMPIRES module since its inception, ${ }^{7}$ to perform PDI in visible $(660-800 \mathrm{~nm})$, sometimes combined with aperture masking for increased resolution. However, until recently, SCExAO was missing the capacity to look at polarized signal in infrared (IR), that HiCIAO used to do. To increase the capabilities of SCExAO to analyze polarized signals, we performed three major upgrades. The first one was to improve the VAMPIRES modules by adding a second science camera for differential imaging, and a fast Ferroelectric Liquid Crystal (FLC). The second upgrade is adding a spectro-polarimetric mode in IR to the CHARIS Integral Field Spectrograph (IFS). ${ }^{8}{ }^{9}$ Finally, the third upgrade focuses on a fast IR PDI mode, similar to VAMPIRES in visible, using a C-RED ONE camera. We present here these three upgrades, and some on-sky results taken during commissioning.

\section{VISIBLE DUAL CAMERA POLARIMETRY WITH VAMPIRES}

The Visible Aperture Masking Polarimetric Imager for Resolved Exoplanetary Structures (VAMPIRES) is one of the visible modules of SCExAO (600-800 nm), available in open-use for astronomers. The goal is to perform high-resolution measurement of the polarization of sources with great accuracy, using various schemes. The

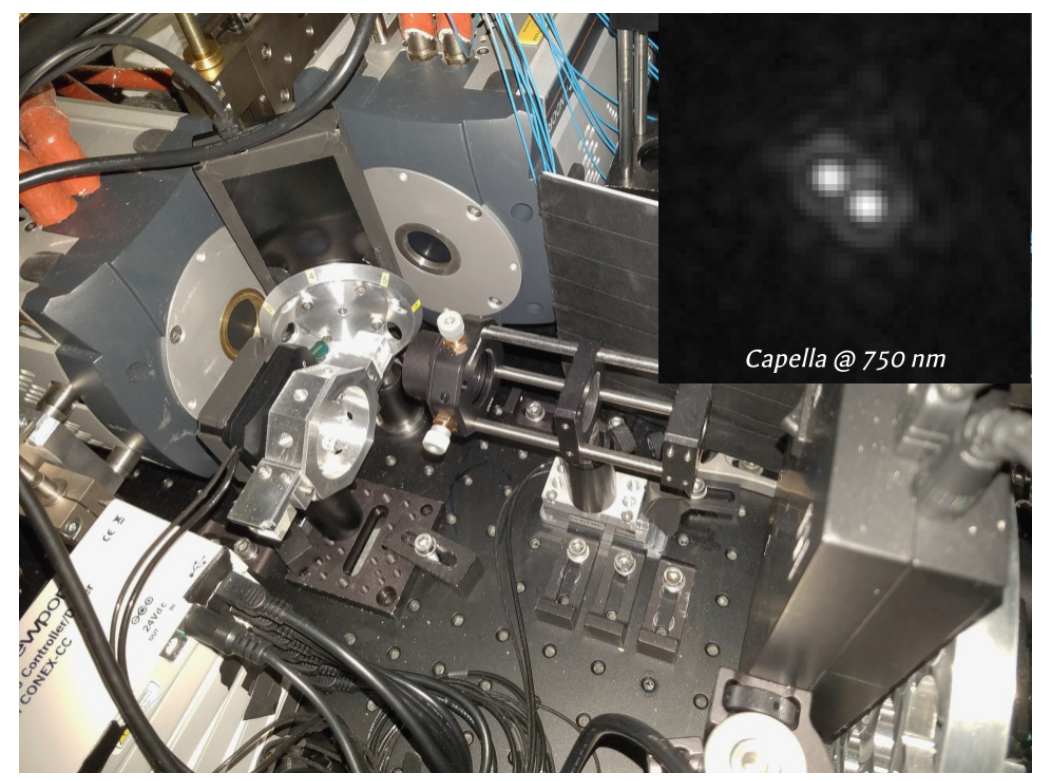

Figure 1. Picture of the VAMPIRES module inside SCExAO. The two cameras are located at the top left, with the differential filter wheel in between, and the beamsplitter cube wheel in front of it. In insert is a picture of the binary Capella, with a separation of 44 mas. 

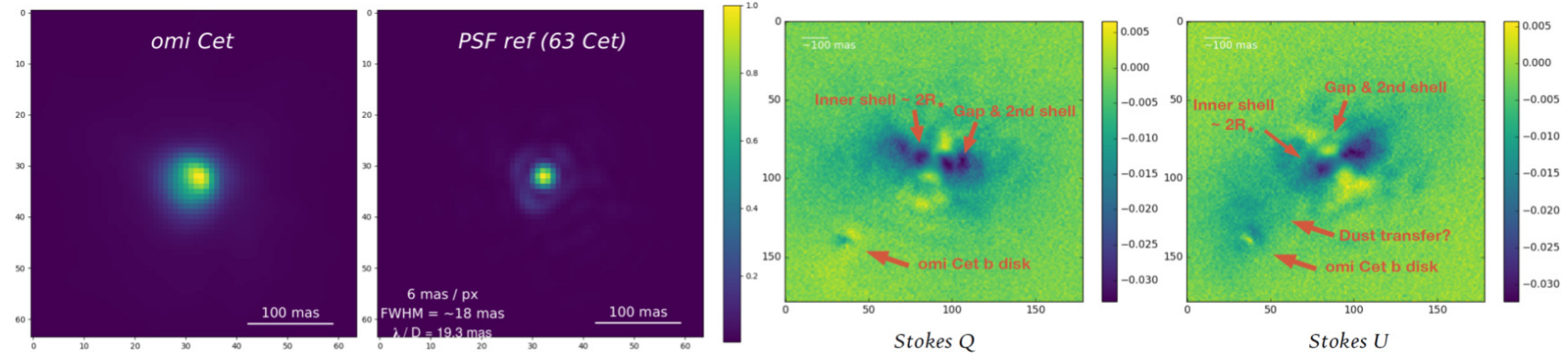

Figure 2. Full intensity image of the resolved star Mira (o Cet) A compared to a reference star, and dust shell in Stokes $\mathrm{Q}$ and $\mathrm{U}$ around Mira A, and disk of dust around the white dwarf companion Mira B. Credit: B. Norris

high-resolution part benefits from the correction of the Pyramid Wavefront Sensor (PyWFS $)^{10}$ to get images with reasonable Strehl ratios $(>20 \%$, see Fig. 1). The instrument can be used in full pupil mode for classical imaging, or in combination with Sparse Aperture Masks (SAM) for known polarized structures — dust shells, binaries, stellar surfaces - for a factor 2 improvement in resolution.

The module went through extensive upgrades in the last few years, to improve the speed and precision of the polarization measurement, as well as new modes. Previously, VAMPIRES used only one camera, and an optical assembly to split both polarizations and image both of them on the detector. The main upgrade was the addition of a second acquisition camera, and a polarizing beamsplitter (see Figure 1). Each polarization is now split with the cube, and imaged with a dedicated camera. This allows to sub-window the acquisition, and run faster than before. To compensate for any differential errors between the two cameras, a Ferroelectric Liquid Crystal (FLC) device acts as a half-wave plate that can be synchronized with the cameras acquisition to flip back and forth the polarization state on each camera. Another half-wave plate, located in front of AO188, is also rotated at a slower rate (typically every minute), to calibrate any polarization errors coming from AO188 and SCExAO.

VAMPIRES has achieved under 10 mas resolution on polarized source, such as the inner mass-loss dust shell around the star $\mu$ Cep, ${ }^{11}$ or the same type of dust-shell around Mira (o Cet) A, as well as a disk around the white dwarf companion Mira B (see the work in progress on Fig. 2 (b)).

The module was also upgraded with a $\mathrm{H}-\alpha$ spectral differential imaging mode, thanks to a motorized mount changing the polarized beamsplitter cube with a 50/50 version, and a differential filter wheel placing a H- $\alpha$ filter in front of one of the cameras, and a continuum filter in front of the other. The filter wheel can then alternate the filters every few minutes. ${ }^{12}$

\section{NIR SPECTRO-POLARIMETRY WITH CHARIS}

Combining adaptive optics and polarimetric differential imaging (PDI) allowed to image dozens of protoplanetary disks composed of gas and dust, notably with HiCIAO on Subaru. With Extreme AO, it is possible to go even closer in inner working angle, and image secondary structures and spirals, as demonstrated by IRDIS on SPHERE, and the polarimetric mode of GPI's integral field Spectrograph (IFS). When HiCIAO was replaced by the IFS CHARIS behind SCExAO, we lost this ability of imaging polarization in NIR. Adding a Wollaston prism inside CHARIS similarly to GPI's IFS, where the Wollaston prism is inserted instead of the dispersing prism, is costly and would replace one of the dispersing modes of CHARIS. Thus we decided instead to add a retractable Wollaston prism at the entrance of CHARIS (room temperature), before the cold stop. In this case, we lose half of the field of view, going from $2 \times 2 \operatorname{arcsec}$ to $1 \times 2 \operatorname{arcsec}$ for each polarization, but we gain the unique capability among high-contrast imagers of performing spectro-polarimetric imaging (See Fig. 3).

The Wollaston prism was designed to split the polarizations evenly with a separation angle of 1 arcsec on sky. A field stop, consisting of a laser-cut rectangular aperture of $2 \times 1$ arcsec in a carbon fiber disk, was installed on a XY translation stage in the last focal plane before the Wollaston prism. This mask removes any cross-talk between polarizations, as illustrated with the lab data of Fig. 4. This figure presents lab measurements taken with SCExAO's polychromatic internal source, a Lyot coronagraph, and CHARIS. Our internal source has very weak flux in K-band, so the tests were performed only in J- and H-band. When the field stop is out (Fig. 4 (a)), 

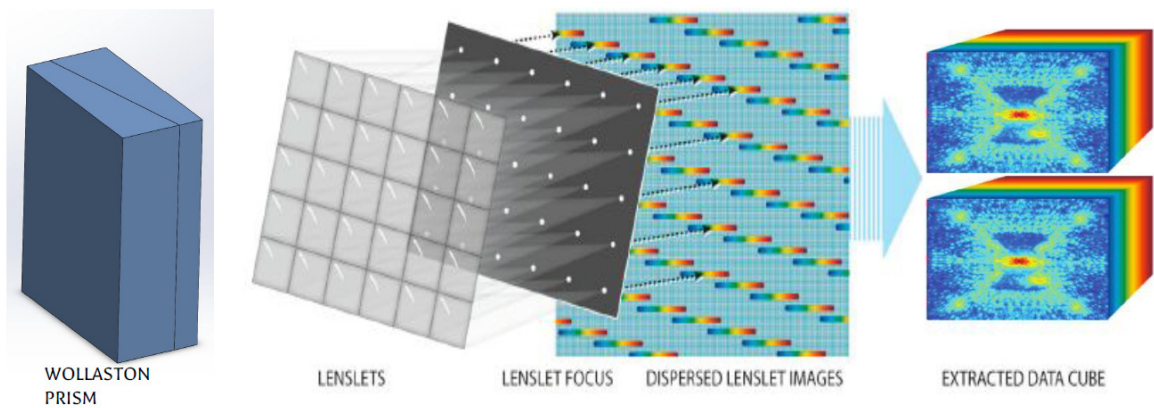

EXTRACTED DATA CUBE

Figure 3. Principle of the spectro-polarimetric mode with CHARIS. The Wollaston splits the two polarizations close to a pupil plane at the entrance of CHARIS, then after entering the Dewar, the light in the focal plane is sampled by a matrix of micro-lenses and an array of pinholes, and dispersed by a prism. The final cube is extracted from the raw image. Each polarization occupy half the normal field of view of CHARIS, i.e. $2 \times 1$ arcsec.

some light from one polarization - here the speckles introduced by the waffle pattern of the DM- can be seen in the other polarization. When the mask is in place (Fig. 4 (b)), this stray light is not visible. Figure 4 (c) and $(\mathrm{d})$ also show that an adaptive speckle grid can still be used for astrometric and photometric calibration, although the regular spacing of $15.9 \lambda / D$ is too large and outside the field of view at the end of H-band (Fig. 4 (c)). Thus a smaller separation of $10 \lambda / D$ can be used (Fig. 4 (d)).

The new PDI mode was successfully tested on-sky during SCExAO's engineering nights in 2019. The polarization was modulated using the half-wave plate in front of AO188, synchronized with the CHARIS acquisitions and the VAMPIRES data cubes (VAMPIRES runs much faster than CHARIS). A good validation of the new PDI mode is presented in Fig. 5, which shows a comparison between the PDI images of AB Aurigae using AO188 and HiCIAO, ${ }^{4}$ VAMPIRES (processing in progress) and the new mode of CHARIS. This preliminary result shows the same complex disk structure, with asymmetric spiral arms, with a slightly smaller inner working angle for the CHARIS image, compared to HiCIAO. In this image, all the wavelengths were collapsed, but with more processing, a spectrum could be extracted. Thanks to a successful commissioning, the spectro-polarimetric mode with CHARIS is now available for Open-Use observers.

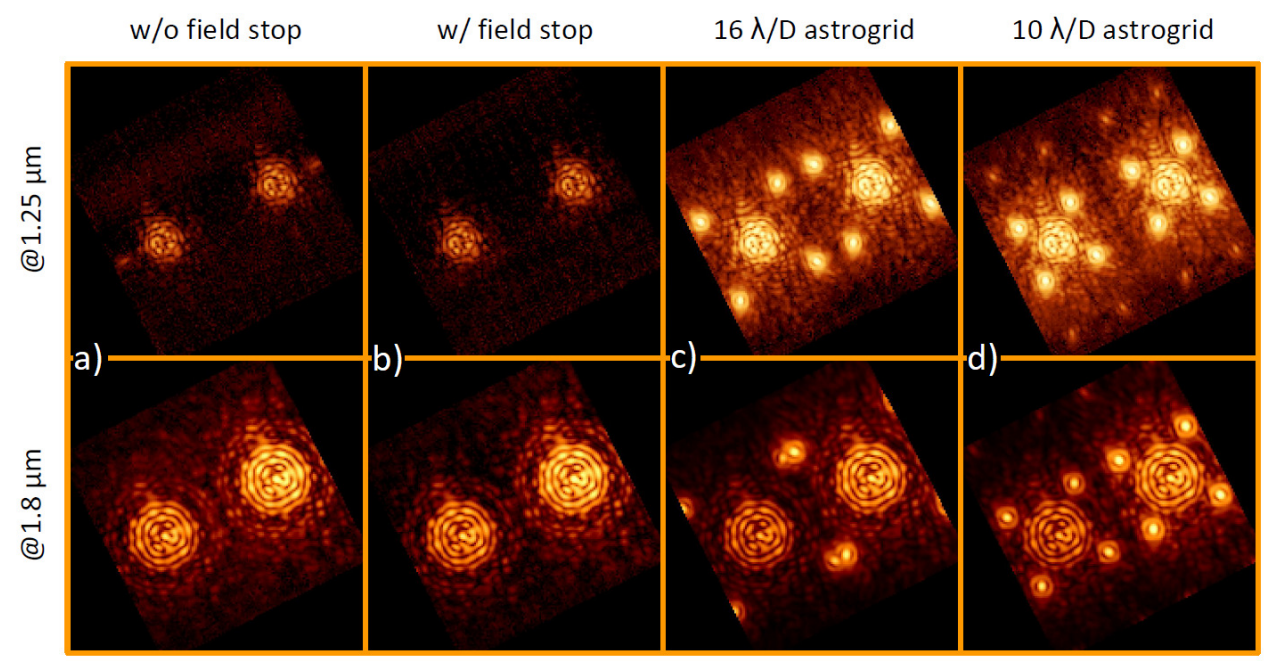

Figure 4. Lab measurements at two different wavelengths (two selected slices in the CHARIS cube), in various conditions. In each case, a Lyot coronagraph with an inner working angle of 113 mas is used. a) Field stop is out, speckles from the quilting of the DM are polluting the polarizations. b) Field stop is in, the quilting speckles are no longer visible. c) Usual speckle grid used with CHARIS, with a separation of $16 \lambda / D$. The separation is too high for the field of view at longer wavelengths. d) A grid with a separation of $10 \lambda / D$ is more adapted to the field of view. 


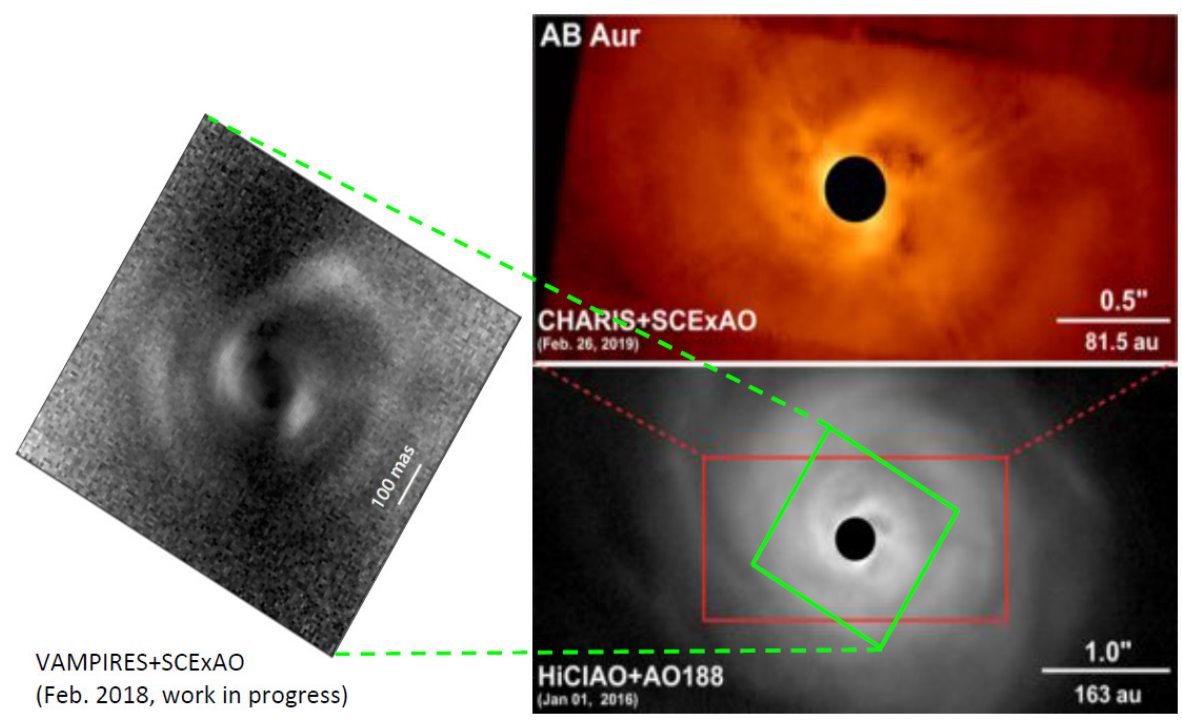

Figure 5. Comparison of the polarimetric intensity images between old HiCIAO observations (2016), and CHARIS observations (2019). In the CHARIS images, the wavelengths were collapsed. The CHARIS image shows a slightly better resolution and inner working angle. In comparison, we also present a VAMPIRES image (work in progress) of the central region. Residual spider diffraction is still visible in the CHARIS image, probably due to uncorrected distortion. Credit: B. Norris and T. Kudo

\section{FAST NIR POLARIMETRY}

The second mode that we implemented on SCExAO uses the latest developments in fast and low-noise IR detectors. Similarly to VAMPIRES in visible, we use a FLC to modulate the polarization at the frequency of the acquisition, while a Wollaston prism, identical to the CHARIS one, splits the polarizations on the detector. The field stop can therefore be shared between CHARIS and this mode, as long as they are oriented the same way. In this case though, the prism can be rotated manually with 45 degree increments. We have therefore four different field stop masks: a horizontal mask, a vertical mask, a 45 deg mask and a 135 deg mask.

In a first step, we used SCExAO's internal NIR camera, a C-RED 2 (see Fig. 6 a)), to perform the fast PDI mode. The C-RED 2 has about 20 times more read noise than the C-RED ONE purchased for the PDI, but it allowed us to rapidly commission this mode while the C-RED ONE was being delivered. We started by testing this mode without the FLC, in broadband mode $(0.95-1.7 \mu \mathrm{m})$, with the same modulation of the polarization
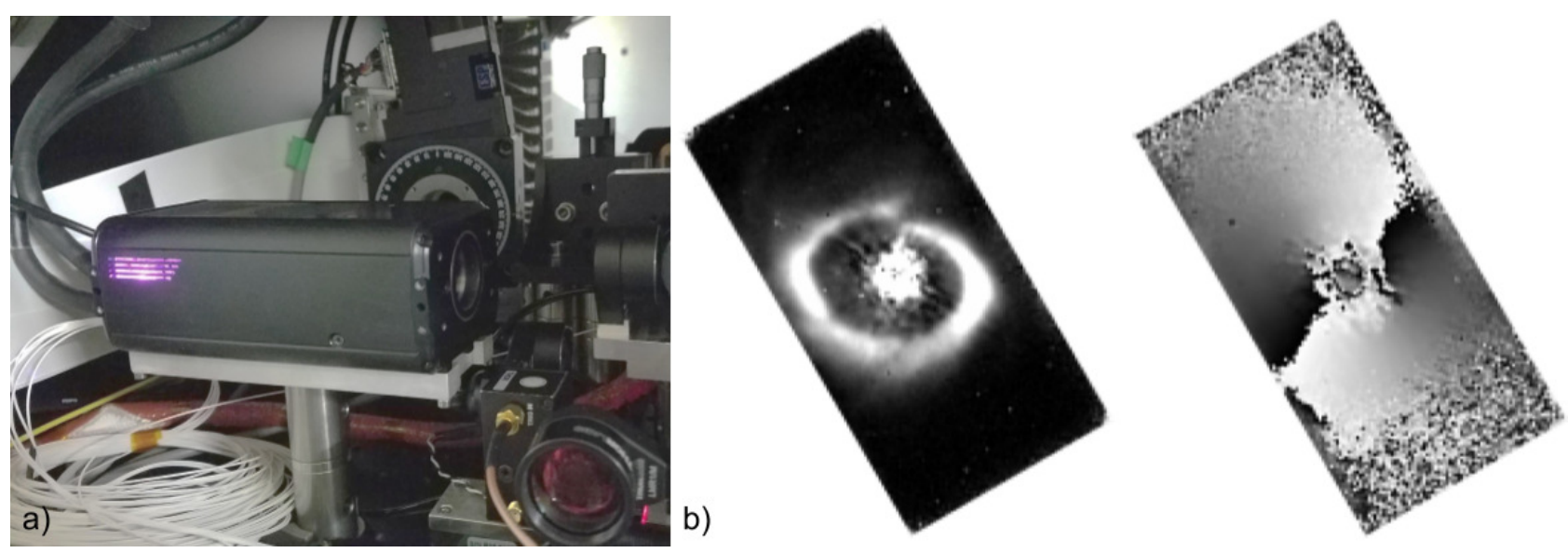

Figure 6. a) Internal C-RED2 camera used for testing of the fast IR polarization mode, b) Polarization intensity and polarization angle of the bright disk around HD34700A, taken in broadband mode $(0.95-1.7 \mu \mathrm{m})$. Credit: J. Zhang 


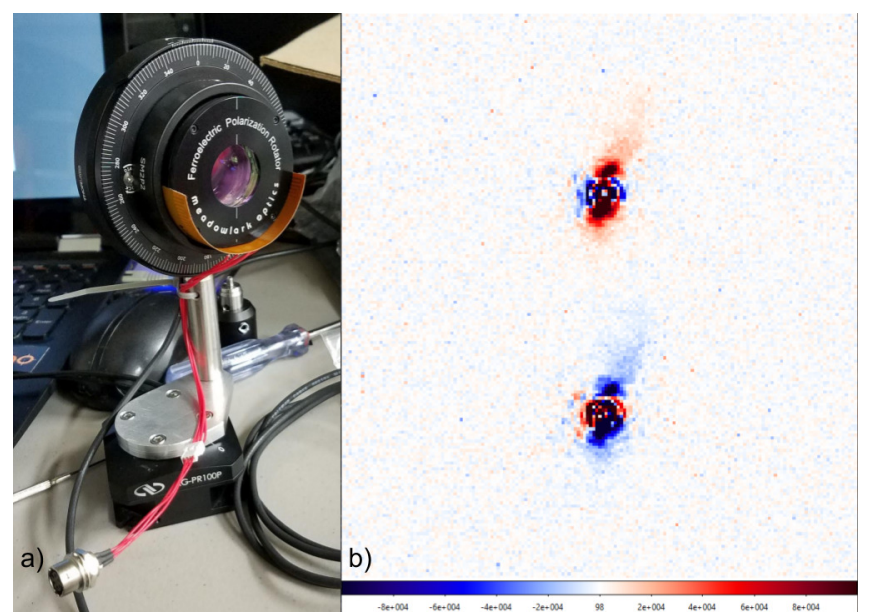

Figure 7. a) FLC installed on a fine rotation mechanism, a heating element to reduce the its switching time, and a mechanism to move the FLC in and out of the beam depending on the configuration. b) Preliminary processing of a few minutes of data on the variable star R Aqr taken at high speed, synchronized with the FLC. A double difference was performed to highlight the dust around the star. Credit: O. Guyon

from AO188's HWP than for CHARIS' PDI mode. Figure 6 b) presents an observation of the bright disk around HD34700A, imaged previously by GPI. ${ }^{13}$ We demonstrated that we can easily detect the polarized signal.

Once the FLC (see Fig. 7 a)) was installed and synchronized with the C-RED 2 camera, we could test the true fast PDI capability of this mode, with frame rates between 1 and $500 \mathrm{~Hz}$. The trigger signal for the FLC and the acquisition are created with offsets to take into account the readout time of the camera and the switching time of the FLC. When the FLC is used, the fast PDI mode is limited to H-band, due to the chromaticity in the retardance of the liquid crystal.

The C-RED ONE camera was integrated to the side of the instrument (see Fig. 8, on the port previously used to test the Institute for Astronomy's SAPHIRA camera. ${ }^{14}$ We started preliminary tests on-sky, although the synchronization with the FLC needs to be completed. We expect this mode to become available for Open-Use observers for the next cycle of proposals.

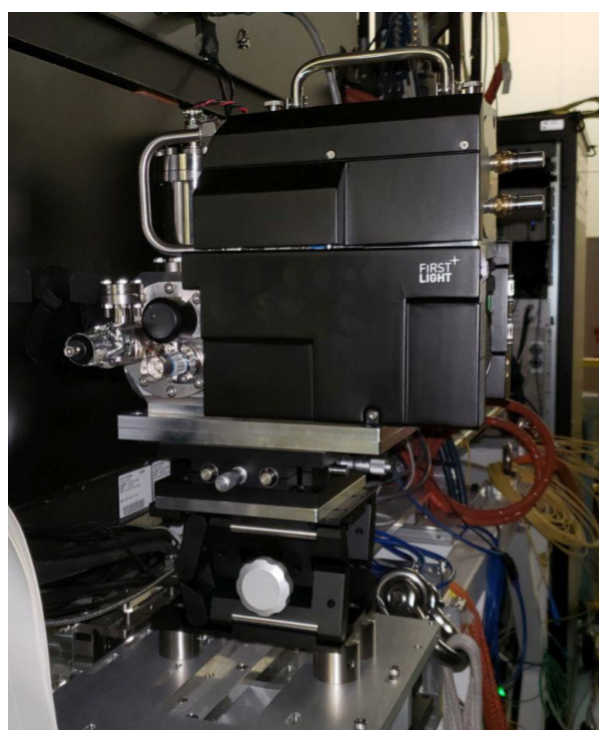

Figure 8. Two fast IR photon-counting camera tested on SCExAO. a) The Kernel camera, a C-RED ONE from First Light Imaging. b) IfA's custom SAPHIRA camera. 


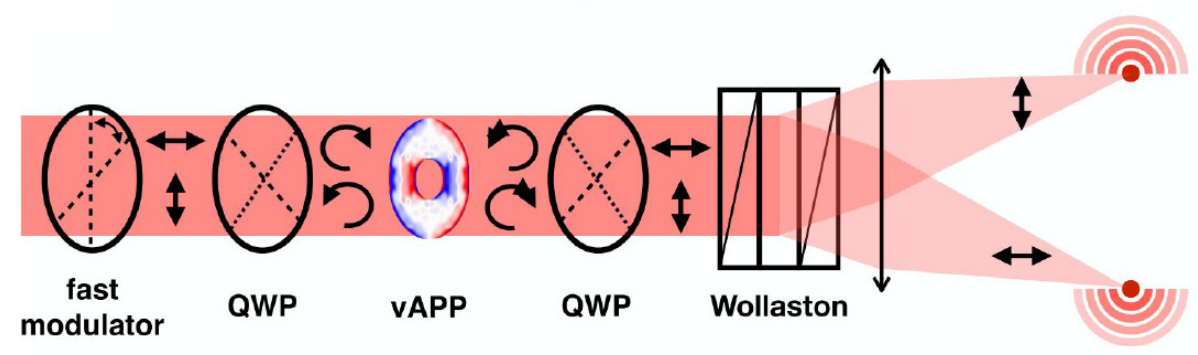

Figure 9. Principle of a polarized vAPP. Credit: S. Bos

\section{FUTURE WORK}

As we mentioned above, the spectro-polarimetric mode with CHARIS is completed and available for Open-Use observations. The fast PDI mode with the C-RED ONE camera is now only missing the synchronization with the FLC, although all the components have already been tested on-sky separately.

Besides this, thanks to the modularity of SCExAO, future upgrades are already scheduled to add new capabilities to these two modes:

- Similarly to VAMPIRES, the IR path will be equipped with non-redundant masks (NRM) to gain in resolution for some specific targets. For the fast-IR polarization mode, masks will be installed in the last pupil plane, between the FLC and the Wollaston prism.

- The same pupil plane can be used to insert a polarimetric vector Apodized Phase Plate (vAPP). ${ }^{15}$ SCExAO is already equipped with a vAPP, where a grating is added to split the two dark zones and image them on CHARIS. The envisioned polarimetric vAPP would not have this grating, the dark zones being separated by the Wollaston prism itself (see Fig. 9). However, the polarimetric vAPP requires circular polarization instead of linear polarization. The mask would then be surrounded by two achromatic quarter-wave plates.

- We are investigating the possibility of measuring the circular polarization of astronomical sources, instead of the linear polarization, using the fast-PDI mode. For this, we can already insert a quarter-wave plate in front of AO188 and synchronize it to the image rotator, to suppress the instrumental polarization of the image rotator. A HWP is then inserted before the Wollaston prism, and rotated to compensate for the rotation of the QWP.

- In a similar way that VAMPIRES was upgraded to perform some $\mathrm{H}-\alpha$ differential imaging, we want to perform some spectral differential imaging with the C-RED ONE camera, this type at the Paschen- $\beta$ line $(1.2822 \mu \mathrm{m})$. The light will be split in two once again with the Wollaston prism, and each beam will go through either the $\mathrm{Pa}-\beta$ filter, or the continuum filter.

\section{CONCLUSION}

Due to its modular design, we can add and improve modules of SCExAO easily and rapidly. One of the most recent improvements were focused mainly on polarization differential imaging, both in visible and infrared. We updated the VAMPIRES module with a second camera and a FLC, allowing for shorter integration times. In addition, two new IR modes were implemented. A spectro-polarimetric mode using the IFS CHARIS, and a fast IR polarimetric mode similar to VAMPIRES, but this time using a C-RED ONE camera.

The spectro-polarimetric mode is now completed, by adding a field stop and a Wollaston prism in front of the IFS CHARIS.This mode is synchronizing the acquisitions of CHARIS and VAMPIRES, since both are sharing AO188's half-wave plate to modulate the polarization state. This mode was successfully tested on-sky, and is now available in Open-Use observations.

the fast PDI mode was tested on-sky using SCExAO's internal NIR camera, while the C-RED ONE camera was integrated on the side of the instrument. The final step to complete this mode is to add the synchronization 
between the C-RED ONE and the FLC, although we already demonstrated this type of synchronization with the C-RED 2 camera. We expect that the fast PDI mode will be available for Open-Use observation for the next cycle of proposals.

Future upgrades include non-redundant masking and polarimetric vAPP, which will allow us to reach lower resolution and higher contrasts that will allow us to probe inner disks and fainter protoplanetary disks. Finally, we are investigating the upgrade of the fast PDI mode with the measure of the circular polarization instead of the linear polarization, and a spectral differential imaging mode at the $\mathrm{Pa}-\beta$ line.

\section{ACKNOWLEDGMENTS}

The development of SCExAO was supported by the Japan Society for the Promotion of Science (Grant-in-Aid for Research \#23340051, \#26220704, \#23103002, \#19H00703 \& \#19H00695), the Astrobiology Center of the National Institutes of Natural Sciences, Japan, the Mt Cuba Foundation and the director's contingency fund at Subaru Telescope. F. Martinache's work is supported by the ERC award CoG - 683029. The authors wish to recognize and acknowledge the very significant cultural role and reverence that the summit of Maunakea has always had within the indigenous Hawaiian community. We are most fortunate to have the opportunity to conduct observations from this mountain.

\section{REFERENCES}

[1] Beuzit, J.-L., Feldt, M., Dohlen, K., Mouillet, D., Puget, P., Wildi, F., Abe, L., Antichi, J., Baruffolo, A., Baudoz, P., Boccaletti, A., Carbillet, M., Charton, J., Claudi, R., Downing, M., Fabron, C., Feautrier, P., Fedrigo, E., Fusco, T., Gach, J.-L., Gratton, R., Henning, T., Hubin, N., Joos, F., Kasper, M., Langlois, M., Lenzen, R., Moutou, C., Pavlov, A., Petit, C., Pragt, J., Rabou, P., Rigal, F., Roelfsema, R., Rousset, G., Saisse, M., Schmid, H.-M., Stadler, E., Thalmann, C., Turatto, M., Udry, S., Vakili, F., and Waters, R., "SPHERE: a 'Planet Finder' instrument for the VLT," in [Ground-based and Airborne Instrumentation for Astronomy II], Proc. Soc. Photo-Opt. Instrum. Eng. 7014, 701418 (July 2008).

[2] Macintosh, B., Graham, J. R., Ingraham, P., Konopacky, Q., Marois, C., Perrin, M., Poyneer, L., Bauman, B., Barman, T., Burrows, A. S., Cardwell, A., Chilcote, J., De Rosa, R. J., Dillon, D., Doyon, R., Dunn, J., Erikson, D., Fitzgerald, M. P., Gavel, D., Goodsell, S., Hartung, M., Hibon, P., Kalas, P., Larkin, J., Maire, J., Marchis, F., Marley, M. S., McBride, J., Millar-Blanchaer, M., Morzinski, K., Norton, A., Oppenheimer, B. R., Palmer, D., Patience, J., Pueyo, L., Rantakyro, F., Sadakuni, N., Saddlemyer, L., Savransky, D., Serio, A., Soummer, R., Sivaramakrishnan, A., Song, I., Thomas, S., Wallace, J. K., Wiktorowicz, S., and Wolff, S., "First light of the Gemini Planet Imager," Proceedings of the National Academy of Science 111, 12661-12666 (Sept. 2014).

[3] Tamura, M., Hodapp, K., Takami, H., Abe, L., Suto, H., Guyon, O., Jacobson, S., Kandori, R., Morino, J.I., Murakami, N., Stahlberger, V., Suzuki, R., Tavrov, A., Yamada, H., Nishikawa, J., Ukita, N., Hashimoto, J., Izumiura, H., Hayashi, M., Nakajima, T., and Nishimura, T., "Concept and science of HiCIAO: high contrast instrument for the Subaru next generation adaptive optics," in [Proc. Soc. Photo-Opt. Instrum. Eng.], 6269, 62690V (Jun 2006).

[4] Hashimoto, J., Tamura, M., Muto, T., Kudo, T., Fukagawa, M., Fukue, T., Goto, M., Grady, C. A., Henning, T., Hodapp, K., Honda, M., Inutsuka, S., Kokubo, E., Knapp, G., McElwain, M. W., Momose, M., Ohashi, N., Okamoto, Y. K., Takami, M., Turner, E. L., Wisniewski, J., Janson, M., Abe, L., Brandner, W., Carson, J., Egner, S., Feldt, M., Golota, T., Guyon, O., Hayano, Y., Hayashi, M., Hayashi, S., Ishii, M., Kandori, R., Kusakabe, N., Matsuo, T., Mayama, S., Miyama, S., Morino, J. I., Moro-Martin, A., Nishimura, T., Pyo, T. S., Suto, H., Suzuki, R., Takato, N., Terada, H., Thalmann, C., Tomono, D., Watanabe, M., Yamada, T., Takami, H., and Usuda, T., "Direct Imaging of Fine Structures in Giant Planet-forming Regions of the Protoplanetary Disk Around AB Aurigae," Astrophys. J. Lett. 729, L17 (Mar 2011).

[5] Jovanovic, N., Martinache, F., Guyon, O., Clergeon, C., Singh, G., Kudo, T., Garrel, V., Newman, K., Doughty, D., Lozi, J., Males, J., Minowa, Y., Hayano, Y., Takato, N., Morino, J., Kuhn, J., Serabyn, E., Norris, B., Tuthill, P., Schworer, G., Stewart, P., Close, L., Huby, E., Perrin, G., Lacour, S., Gauchet, L., Vievard, S., Murakami, N., Oshiyama, F., Baba, N., Matsuo, T., Nishikawa, J., Tamura, M., Lai, O., Marchis, F., Duchene, G., Kotani, T., and Woillez, J., "The Subaru Coronagraphic Extreme Adaptive 
Optics System: Enabling High-Contrast Imaging on Solar-System Scales," Pub. Astron. Soc. Pacific 127, 890 (Sep 2015).

[6] Lozi, J., Guyon, O., Jovanovic, N., Goebel, S., Pathak, P., Skaf, N., Sahoo, A., Norris, B., Martinache, F., N'Diaye, M., Mazin, B., Walter, A. B., Tuthill, P., Kudo, T., Kawahara, H., Kotani, T., Ireland, M., Cvetojevic, N., Huby, E., Lacour, S., Vievard, S., Groff, T. D., Chilcote, J. K., Kasdin, J., Knight, J., Snik, F., Doelman, D., Minowa, Y., Clergeon, C., Takato, N., Tamura, M., Currie, T., Takami, H., and Hayashi, M., "SCExAO, an instrument with a dual purpose: perform cutting-edge science and develop new technologies," in [Proc. Soc. Photo-Opt. Instrum. Eng.], Society of Photo-Optical Instrumentation Engineers (SPIE) Conference Series 10703, 1070359 (Jul 2018).

[7] Norris, B., Schworer, G., Tuthill, P., Jovanovic, N., Guyon, O., Stewart, P., and Martinache, F., "The VAMPIRES instrument: imaging the innermost regions of protoplanetary discs with polarimetric interferometry," Mon. Not. R. Astr. Soc. 447, 2894-2906 (Mar 2015).

[8] McElwain, M. W., Brandt, T. D., Janson, M., Knapp, G. R., Peters, M. A., Burrows, A. S., Carlotti, A., Carr, M. A., Groff, T., Gunn, J. E., Guyon, O., Hayashi, M., Kasdin, N. J., Kuzuhara, M., Lupton, R. H., Martinache, F., Spiegel, D., Takato, N., Tamura, M., Turner, E. L., and Vanderbei, R. J., "Scientific design of a high contrast integral field spectrograph for the Subaru Telescope," in [Proc. Soc. Photo-Opt. Instrum. Eng.], Society of Photo-Optical Instrumentation Engineers (SPIE) Conference Series 8446, 84469C (Sep 2012).

[9] Groff, T. D., Peters, M. A., Kasdin, N. J., Knapp, G., Galvin, M., Carr, M., McElwain, M. W., Brandt, T., Janson, M., Gunn, J. E., Lupton, R., Guyon, O., Martinache, F., Jovanovic, N., Hayashi, M., and Takato, N., "Design of the CHARIS integral field spectrograph for exoplanet imaging," in [Techniques and Instrumentation for Detection of Exoplanets VI], Proc. Soc. Photo-Opt. Instrum. Eng. 8864, 88640H (Sept. 2013).

[10] Lozi, J., Jovanovic, N., Guyon, O., Chun, M., Jacobson, S., Goebel, S., and Martinache, F., "Visible and Near Infrared Laboratory Demonstration of a Simplified Pyramid Wavefront Sensor," Pub. Astron. Soc. Pacific 131, 044503 (Apr 2019).

[11] Norris, B. R. M., Tuthill, P. G., Ireland, M. J., Lacour, S., Zijlstra, A. A., Lykou, F., Evans, T. M., Stewart, P., and Bedding, T. R., "A close halo of large transparent grains around extreme red giant stars," Nature (London) 484, 220-222 (Apr 2012).

[12] Uyama, T., Norris, B., Jovanovic, N., Lozi, J., Tuthill, P., Guyon, O., Kudo, T., Hashimoto, J., Tamura, M., and Martinache, F., "High-contrast $\mathrm{H} \alpha$ imaging with Subaru/SCExAO+VAMPIRES," arXiv e-prints , arXiv:2008.10780 (Aug. 2020).

[13] Monnier, J. D., Harries, T. J., Bae, J., Setterholm, B. R., Laws, A., Aarnio, A., Adams, F. C., Andrews, S., Calvet, N., Espaillat, C., Hartmann, L., Kraus, S., McClure, M., Miller, C., Oppenheimer, R., Wilner, D., and Zhu, Z., "Multiple Spiral Arms in the Disk around Intermediate-mass Binary HD 34700A," Astrophys. J. 872, 122 (Feb 2019).

[14] Goebel, S. B., Hall, D. N. B., Guyon, O., Warmbier, E., and Jacobson, S. M., "Overview of the SAPHIRA detector for adaptive optics applications," Journal of Astronomical Telescopes, Instruments, and Systems 4, 026001 (Apr 2018).

[15] Bos, S. P., Doelman, D. S., de Boer, J., Por, E. H., Norris, B., Escuti, M. J., and Snik, F., "Fully broadband vAPP coronagraphs enabling polarimetric high contrast imaging," in [Proc. Soc. Photo-Opt. Instrum. Eng.], Society of Photo-Optical Instrumentation Engineers (SPIE) Conference Series 10706, 107065M (Jul 2018). 